\title{
Interaction of low-energy electrons with surface polarity near ferroelastic domain boundaries
}

\author{
Z. Zhao, ${ }^{1}$ N. Barrett, ${ }^{2}$ Q. Wu, ${ }^{2}$ D. Martinotti, ${ }^{2}$ L. Tortech, ${ }^{3,4}$ R. Haumont,${ }^{5}$ M. Pellen, ${ }^{5}$ and E. K. H. Salje ${ }^{6,7, *}$ \\ ${ }^{1}$ School of Materials Science and Engineering, Xi'an University of Technology, Xi'an 710048, China \\ ${ }^{2}$ SPEC, CEA, CNRS, Université Paris-Saclay, CEA Saclay, 91191 Gif-sur-Yvette Cedex, France \\ ${ }^{3}$ Sorbonne Université, CNRS, Institut Parisien de Chimie Moléculaire, IPCM, 4 Place Jussieu, F-75005 Paris, France \\ ${ }^{4}$ LICSEN, NIMBE, CEA, CNRS, Université Paris-Saclay, CEA Saclay, 91191 Gif-sur-Yvette Cedex, France \\ ${ }^{5}$ Laboratoire de Physico-Chimie de l'Etat Solide, ICMMO, CNRS-UMR 8182, Bâtiment 410, Université Paris-Sud XI, \\ 15 rue Georges Clémenceau, 91405 Orsay Cedex, France \\ ${ }^{6}$ Department of Earth Sciences, University of Cambridge, Downing Street, Cambridge CB2 3EQ, United Kingdom \\ ${ }^{7}$ State Key Laboratory for Mechanical Behavior of Materials, Xi'an Jiaotong University, Xi'an 710049, China
}

(Received 20 December 2018; published 1 April 2019)

\begin{abstract}
We derive surface polarity at and near ferroelastic domain boundaries from molecular dynamics simulations based on an ionic spring model. Interatomic gradient forces lead to flexoelectricity which, in turn, generates polarity at the surface and in twin boundaries. We then derive generic properties of electron scattering spectra equivalent to those observed in low-energy electron microscopy (LEEM) and mirror electron microscopy (MEM) experiments. Negatively (positively) charged surfaces reflect (attract) incident electrons with low kinetic energy. The electron images reveal the valley and ridge surface structures near the intersection of the twin boundary and the surface. Polarity in surface layers is predicted to be visible in LEEM and MEM spectra at neutral surfaces, but much less when surfaces are charged. Inward polarity reflects electrons similar to negative surface charges, and outward polarity backscatters electrons like positive surface charges. Both the polarity in the twin boundary and the physical topography scatter electrons, consistent with experimental LEEM and MEM experiments on $\mathrm{CaTiO}_{3}$ with (001) and (111) surface terminations.
\end{abstract}

DOI: 10.1103/PhysRevMaterials.3.043601

\section{INTRODUCTION}

Ferroelastic materials display domain structures [1,2], which carry polarity at domain boundaries [3] and near surfaces [4]. Twin boundaries are very narrow regions, typically a few nanometers thick [5,6], where the spontaneous strain changes sign [7]. Large strain gradients exist in ferroelastic twin boundaries and generate novel properties inside the boundaries and near surfaces [8-11]. These "emerging functionalities" are absent in the bulk $[12,13]$.

The functionality of twin boundaries has been intensively studied during the last decade. For instance, ferroelastic twins can be polar [3,14-17], superconducting [18,19], or even chiral $[20,21]$. The ferroelectricity of twin boundaries in $\mathrm{CaTiO}_{3}$ $[3,14]$ and $\mathrm{SrTiO}_{3}[16]$ is particularly important because computer simulations have predicted that the polarization can be switched by weak electric fields [22]. For this reason, ferroelastic materials with ferroelectric twin boundaries have potential applications as memory devices with ultrahigh storage density and lower power consumption. The emergence of polarity in the twins was theoretically interpreted as coupling of order parameters in topologically singular regions so that polarization disappears outside the twin boundary $[20,21]$. Polarity is also known to exist at surfaces [23-26], although this effect has been much less studied than polarity in domain boundaries. It was found by first-principles studies

\footnotetext{
*Corresponding author: ekhard@esc.cam.ac.uk
}

that surface relaxation can induce polarity in an otherwise nonpolar matrix of $\mathrm{CaTiO}_{3}[25,26]$ and $\mathrm{SrTiO}_{3}[23,24]$. The relaxations are shown to depend sensitively on the terminating atoms in the surface, and can enhance or reduce the surface polarization.

Ferroelastic surfaces are modified for another reason [27-29]. Novak and Salje studied the distribution of lattice strain near the intersection of surface layers and twin boundaries $[27,28]$. They found that twin boundaries close to the surface show equal strain curves with a groove profile and two ridges, one on each side of the twin boundary. Such strain profiles are then expected to generate local polarization via the flexoelectric (or other) coupling effects [29].

Experimentally, the intersection of twin boundaries with the surface of $\mathrm{CaTiO}_{3}$ was studied by low-energy electron microscopy (LEEM) [30]. This technique provides full-field, noncontact imaging of surface potential with a spatial resolution better than $20 \mathrm{~nm}$ [31]. Incident electrons with very low kinetic energy are reflected before reaching the surface [using mirror electron microscopy (MEM)], while the electrons with higher energy can penetrate the sample surface and are elastically backscattered (LEEM) [31-34]. The transition between MEM and LEEM provides a direct measure of the surface potential. MEM images of the surface of $\mathrm{CaTiO}_{3}$ show contrast between twin boundaries and the domains, indicating polarity of the twin boundaries with respect to the nonpolar bulk. However, MEM and LEEM images are very sensitive not only to polarity but also to other factors, including surface topography and charge. Twin boundaries can have a 
factory rooflike topography and surface terminations can be charged. Hence, the relation between surface polarity at the twin boundary and the surface potential provided by the MEM technique is rather ill defined. Furthermore, careful consideration of the electron optics used for imaging is required in order to correctly interpret observed experimental contrast. For example, a negative point charge on the surface appears dark if imaged directly or with an even number of converging lenses, but bright if the imaging optics contain an odd number of lenses [34,35].

In this paper, we present the results of large-scale molecular dynamics simulation of the interaction of low-energy electrons with ferroelastic surfaces containing twin walls, in order to simulate MEM and LEEM experiments. Physical topography, charge, and surface dipoles all play a role in determining the electron intensity distribution measured in MEM and LEEM experiments. The simulations are compared with experimental data recorded on $\mathrm{CaTiO}_{3}$ (001) and (111) surfaces, containing different twin walls and displaying uncharged or charged terminations. Charge dominates polarity in determining the image contrast but, in the case of nominally neutral surfaces, polarity or physical topography takes over. In the absence of physical topography, vortexlike rotation of the surface polarity can provide subtle effects in the electron intensity, weaker but similar to those observed at the surface of ferroelectric domain boundaries.

\section{METHODS}

\section{A. Simulations}

The simulation is based on a two-dimensional (2D) ionic spring model $[29,36]$. This "standard model" was designed for molecular dynamics (MD) simulations to explore polar effects in complex ferroelastic domain patterns with a large number of particles (typically, $10^{5}-10^{6}$ atoms) and long MD running times. The model contains two sublattices. In the anion sublattice, all ions have negative elementary charge $\left(-1.602 \times 10^{-19} \mathrm{C}\right)$, whereas ions in the cation sublattice have positive elementary charge. The sublattices have an equal number of ions to ensure the electric neutrality of the system. Short-range interatomic potentials are designed separately for each sublattice. All energies for the structural relaxation are in relative units; all distances are lattice units. In the anion sublattice, harmonic springs are used for the firstnearest $\left[U(\mathbf{r})=20(\mathbf{r}-1)^{2}\right.$, black in Fig. 1(a) $]$ and the thirdnearest neighbor $\left[U(\mathbf{r})=8(\mathbf{r}-2)^{4}\right]$, where $\mathbf{r}$ is the lattice distance vector. Landau-type double-well potentials $[U(\mathbf{r})=$ $-10(\mathbf{r}-\sqrt{2})^{2}+8000(\mathbf{r}-\sqrt{2})^{4}$, gray sticks along diagonal in Fig. 1(a)] applied to the second-nearest neighbors generate unit cells with a $2^{\circ}$ shear angle. This mimics the single crystal (embedded in a three-dimensional matrix) undergoing a tetragonal $4 / \mathrm{mmm}$ to orthorhombic $\mathrm{mmm}$ phase transition. As a result, the anion sublattice forms ferroelastic twin structures, but the unit cells remain centrosymmetric as in $\mathrm{CaTiO}_{3}$ with cations located at the center of the anion unit cells. The interactions between cations are described by harmonic springs where only the first-nearest neighbors $\left[U(\mathbf{r})=20(\mathbf{r}-1)^{2}\right.$, green in Fig. 1(a)] and the second-nearest neighbors $[U(\mathbf{r})=$ $\left.1.5(\mathbf{r}-\sqrt{2})^{2}\right]$ are considered. The sublattices are coupled by
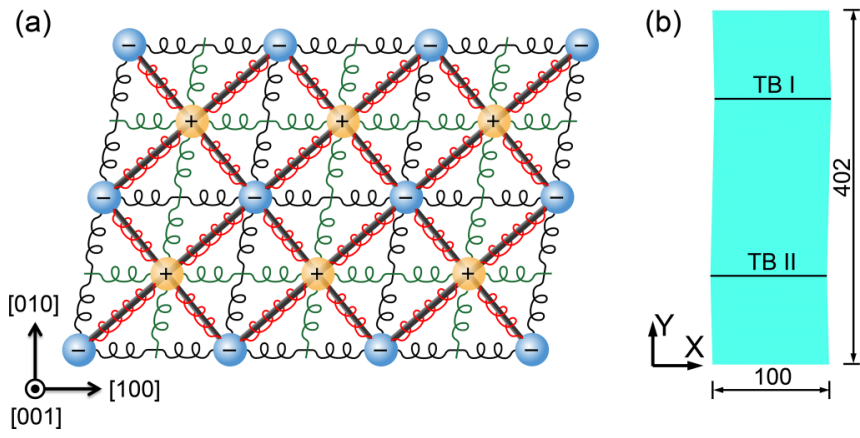

FIG. 1. (a) Sketch of a small section of the model with two ionic sublattices for $2 \times 3$ lattice units. Coulomb interactions and interatomic interactions combine in the model. The interatomic interactions between the nearest neighbors are harmonic (shown by springs). The anions are blue and the cations are orange. Double-well potentials (gray sticks) along diagonals in the ferroelastic anion sublattice lead to the formation of twin structures. (b) The complete simulated cell has two twin boundaries. The valley intersection is the upper intersection and the ridge configuration is the lower intersection [29]. The left-hand side of the slab is considered to be the surface.

harmonic springs $\left[U(\mathbf{r})=0.5(\mathbf{r}-\sqrt{2} / 2)^{2}\right.$, red in Fig. 1(a) $]$. Long-range Coulomb interactions are superimposed on the entire model and a dielectric constant of 1000 is adopted to balance the long-range interactions and short-range interactions. The anharmonic component driving the development of the microstructure is hence located in the anion lattice alone; all secondary microstructures are induced by the interlattice coupling [29]. The complete sample is configured with two preexisting horizontal domain boundaries [Fig. 1(b)] and has dimensions $100 \times 402$ lattice units. The twin boundaries are labeled TB I and TB II in Fig. 1(b). Only the left-hand layers of the sample are considered in this paper; all other configurations follow by symmetry. Domain boundary I shows a valley configuration and domain boundary II shows a ridge in the left surface layer. Periodic boundary conditions were adopted in the $y$ direction and open boundary conditions were adopted in the $x$ direction (direction of the surface normal). We relax the sample at very low temperature $\left(0.001 T_{\mathrm{C}}\right)$ to avoid thermally induced disorder (see Ref. [36] for comparison with thermal excitations). The relaxation generates the local dipole moments in the surface and along the twin boundaries. Once the structural relaxations are simulated, they are frozen. No further relaxation is considered when electrons are scattered by these structures.

We studied structures with cation, anion, and charge neutral (equal numbers of cations and anions) surface terminations. The model dipoles are defined by vectors pointing from the center of negative charge towards the positive charge in each unit cell, i.e., it is a point charge model. Given the centrosymmetric bulk structure, the net dipole strength in the bulk is zero but becomes nonzero near the twin boundaries and the surface. We then simulated the scattering of low-energy electrons at the surface. The incident electrons were created with random coordinates in a region in the vacuum to the left of the sample. They move toward the surface with a given initial kinetic energy. Only Coulomb interactions between electrons 
and the sample are considered. Interactions between electrons were ignored so that each electron is scattered independently. This is a reasonable assumption for LEEM experiments using beam currents below the critical value for the development of space charge effects. A virtual screen was set at a distance of 50 lattice units to the left of the sample. When an electron is reflected before penetrating the sample surface (MEM) or backscattered by the surface region (LEEM), it moves to the left and is detected at the screen. The electron distribution represents the brightness contrast in the MEM/LEEM image due to electron-ion interactions. Note that the point charge nature of the model means that the interaction between the incident electrons and the electron clouds of the cations and anions is not taken into account, only that between the electrons and the net elementary charge of the ions. There is therefore no quantity which can be directly related to the surface potential; however, this simple model does allow one to directly study the effects of charge and polarity on the observed contrast in electron images. All simulations were performed using the computer code LAMMPS [37]; NPT ensemble was used in the isothermal and isobaric simulations. The temperature and pressure of the sample were held constant by the Nosé-Hoover thermostat and barostat [38,39]; the visualization was done with the ATOMEYE code [40].
Our model contains only cations and anions in a $\mathrm{NaCl}$ configuration. We consider the termination of a crystal as either positive (all cations), negative (all anions), or neutral when there is an equal number of cations and anions in a checkerboard configuration. Other structure types or other surfaces in our model contain different mixtures of cations and anions but do not fundamentally change the electron-ion interactions studied here. Let us take the perovskite structure as an example where different charge states are found on different surfaces. The probable charged surface layers have been discussed in Refs. [23,24]. Our generic results can then be scaled with respect to the actual surface charge for any specific structure type.

\section{B. Experiment}

We compare our simulation results with experimental observation of ferroelastic $\mathrm{CaTiO}_{3} \cdot \mathrm{CaTiO}_{3}$ is a system where no polarity exists in the bulk, but where the twin boundaries are known to be polar.

The $\mathrm{CaTiO}_{3}$ single crystals for LEEM and MEM experiments were made at ICMMO (Paris Sud) and cut along (001) and (111) faces. The three possible ionic terminations in the model allow comparison with experimental MEM-LEEM
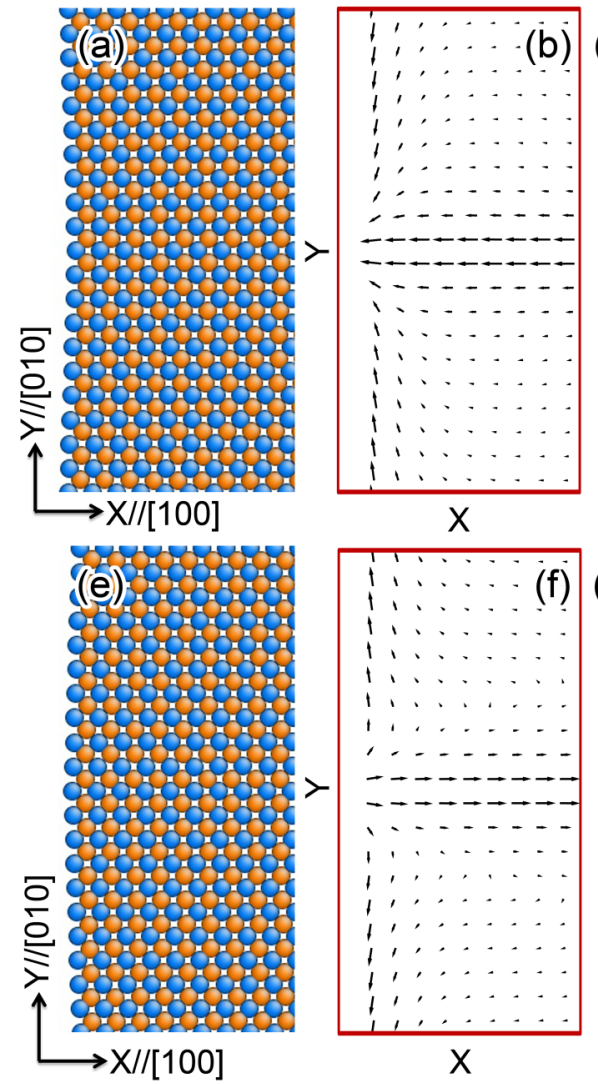
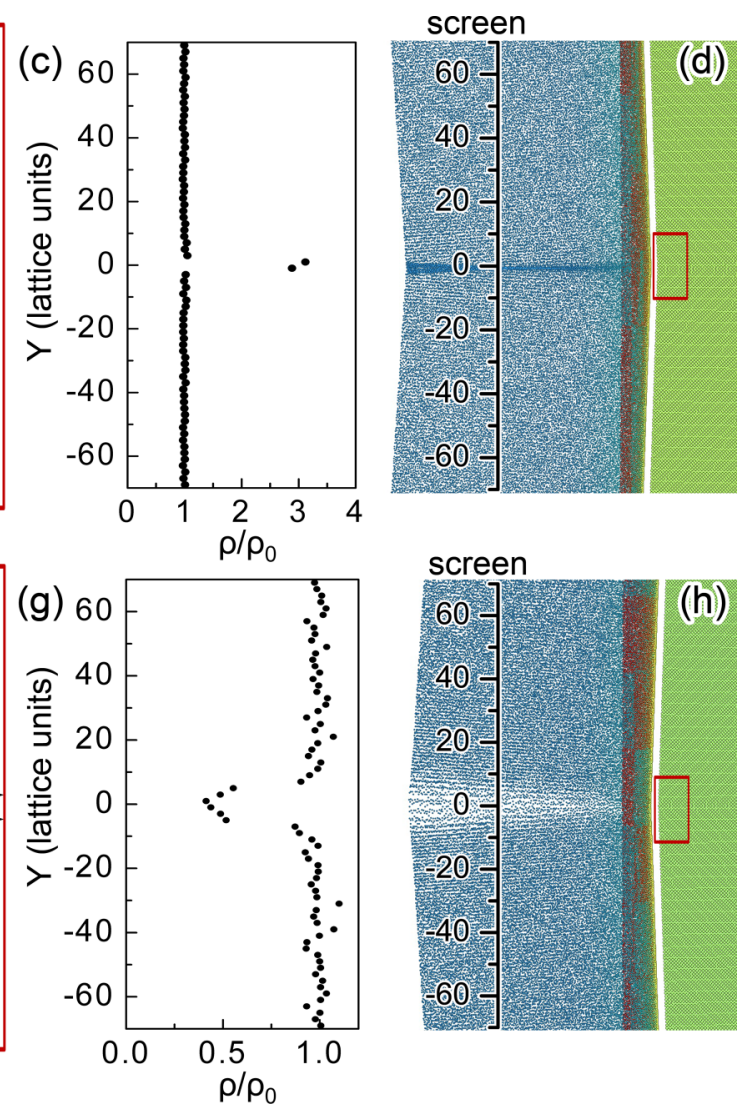

FIG. 2. Polarity at anion-terminated surfaces. (a), (b) The atomic configurations and polarizations near twin boundary I (a valley). The dipole displacement is amplified by a factor of 25 for clarity. Cations are colored in orange and anions are colored in blue. (d) A snapshot illustrates the incident electrons being reflected by the surface. Red electrons are approaching the surface and blue electrons are departing from the surface (the mixture gives rise to the gray color of the electrons). The red rectangle in the green sample indicates the region shown in (a) and (b). The reduced density $\left(\rho / \rho_{0}\right)$ of electrons projected on the virtual screen is shown in (c), $\rho_{0}$ is the electron density in the initial configuration. (e)-(h) are the corresponding results for twin boundary II (a ridge). 
results on $\mathrm{CaTiO}_{3}(001)$, which is charge neutral whether $\mathrm{CaO}$ or $\mathrm{TiO}_{2}$ terminated, and $\mathrm{CaTiO}_{3}$ (111), which has negative $\left(\mathrm{CaO}_{3}{ }^{4-}\right)$ or positive $\left(\mathrm{Ti}^{4+}\right)$ termination. They underwent 5 min ozone exposure in air to remove carbon-based surface contamination and were then introduced into to the ElmitecIII MEM-LEEM ultrahigh vacuum (UHV, base pressure $2 \times$ $10^{-8} \mathrm{~Pa}$ ) system. Surface crystallography and sample orientation were checked by low-energy electron diffraction (LEED) in the LEEM. Electron images were acquired at $300^{\circ} \mathrm{C}$ to minimize charging and in a field of view $75 \mu \mathrm{m}$. The spatial resolution was $15 \mathrm{~nm}$. The incident electron energy, also called the start voltage (SV), is defined by the sample bias with respect to the work function of the electron gun.

\section{RESULTS}

\section{A. Simulations}

In our 2D model, the (100) plane is charged. The topography of the relaxed anion-terminated surfaces contains valleys and ridges at the intersections with twin boundaries. We will therefore compare with the $\mathrm{CaO}_{3}^{4-}$ surface termination of $\mathrm{CaTiO}_{3}$ (111). Twin boundary I generates a valley in the surface [Fig. 2(a)]. Far from the surface, the strain gradient in twin boundary I produces dipoles pointing to the left, i.e., out of the surface, while the bulk remains nonpolar [Fig. 2(b)]. In the first layers, polarity appears in the twin boundary and extends over a large section of the surface. The surface dipoles point to the twin boundary and slightly outwards. The twin boundary II intersection with the surface is a ridge [Fig. 2(e)]. The dipoles in the twin boundary point to the right [Fig. 2(f)]. Polarity appears in the surface layers with dipoles pointing away from the twin boundary and slightly outwards. In both cases (ridge or valley), polarity appears in the twin boundary and at the surface. The component of the surface dipole perpendicular to the surface appears independent of the twin topography and polarity and is constant over long distances. The surface dipoles are mirror symmetric with respect to the twin boundary.

When incident electrons with low initial kinetic energy of $33 \mathrm{meV}$ approach the surface, they are reflected by Coulomb interaction with negatively charged ions. The white strip between the electrons and the sample surface in Figs. 2(d) and 2(h) indicates that they are indeed reflected before reaching the surface, simulating the MEM condition, although the reflection is due purely to the Coulomb interaction with the negative point charges. The reflected electrons recorded on the virtual screen are focused by the valley but scattered by the ridge [Figs. 2(d) and 2(h)]. The screen is positioned at the focal point of the valley twins, 50 u.c. from the surface. Screens placed further from the surface show lower intensity because they are beyond the focal point. Therefore, the electron density near a twin boundary is different from that far from the twin boundary, namely, a valley surface generates higher electron intensities at the twin boundary while ridge
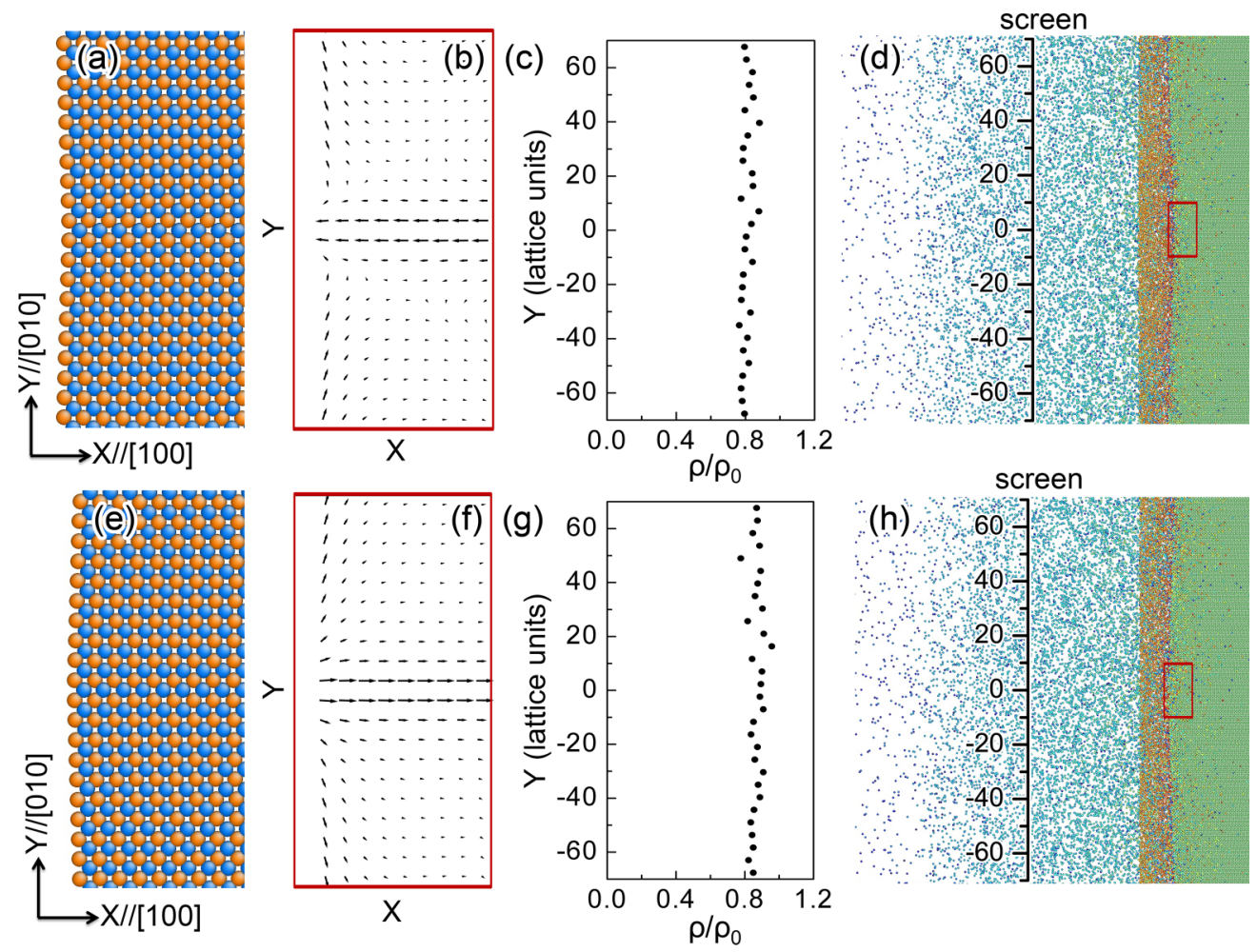

FIG. 3. Polarity at cation-terminated surfaces. (a), (b) The atomic configurations and dipoles near twin boundary I. The dipole displacement is amplified by a factor of 25 for clarity. The color code is as in Fig. 2. (d) A snapshot illustrates the incident electrons being reflected by the surface. Red electrons are approaching the surface and blue electrons are departing from the surface. The red rectangle indicates the region of (a) and (b). Most electrons penetrate the surface, attracted by the positive surface charge. The virtual screen is indicated by the solid line. The reduced density $\left(\rho / \rho_{0}\right)$ of electrons projected on the virtual screen at $Y$ positions with respect to the twin boundary is shown in (c); $\rho_{0}$ is the electron density in the initial configuration. (e)-(h) are the corresponding results for twin boundary II (ridge). 

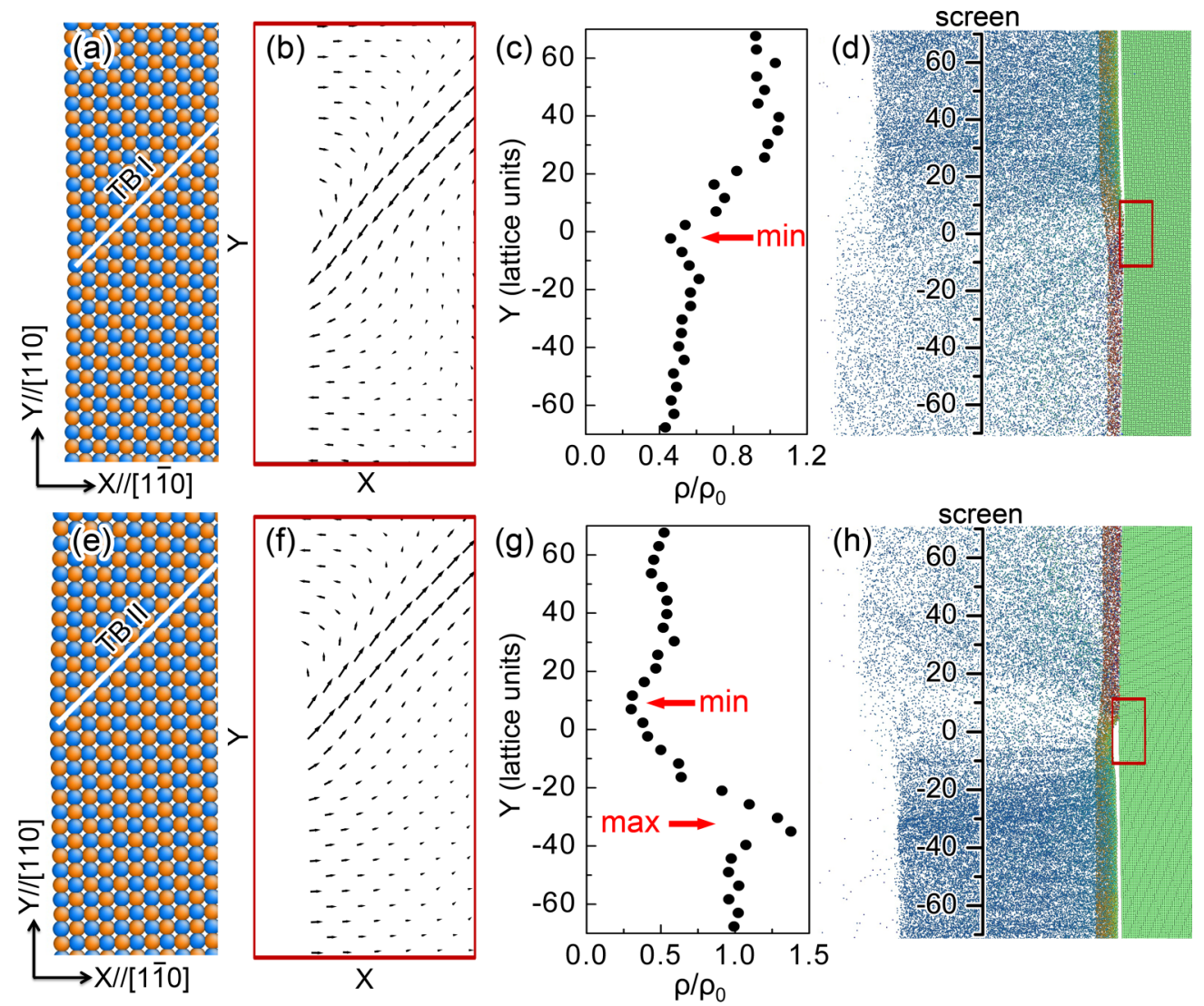

FIG. 4. Polarity at neutral terminations with twin walls at $45^{\circ}$ with respect to surface. (a), (b) The atomic configurations and corresponding dipole polarizations near twin boundary I (shallow valley). The dipole displacement is amplified by a factor of 25 for clarity. Cations are colored in orange and anions are colored in blue in (a). (d) A snapshot illustrates the incident electrons being reflected by the surface. Red electrons are approaching the surface and blue electrons are departing from the surface. The red rectangle in the green sample shows the region where (a) and (b) are extracted. The reduced density $\left(\rho / \rho_{0}\right)$ of electrons projected on the virtual screen at $Y$ positions with respect to the twin boundary is shown in (c); $\rho_{0}$ is the electron density in the initial configuration. (e)-(h) are the corresponding results for twin boundary II (shallow ridge).

surface generates lower electron intensities [Figs. 2(c) and $2(\mathrm{~g})]$. The absolute value of the change in intensity in the image of the twin is not the same for valleys and ridges with respect to that reflected by the flat surface. This is because the valley reflects electrons from two inclined planes pointing towards the position of the twin, whereas the ridge scatters electrons in two directions pointing away from each other. The domain surfaces on either side of both twins show positive (outward pointing) dipoles. However, the electrons are still reflected before reaching the surface, showing that charge dominates over dipoles.

The polarity at cation-terminated surfaces is fundamentally different from anion-terminated surfaces (Fig. 3). This can be considered close to the case of $\mathrm{Ti}^{4+}$, i.e., positively charged, termination of $\mathrm{CaTiO}_{3}$ (111). Start energy of $33 \mathrm{meV}$ is used. The positive ions attract the electrons and accelerate them into the surface. As can be seen in Figs. 3(d) and 3(h), there is no white stripe characteristic of electron reflection, but a continuum of incident electrons penetrating the surface. The electrons recorded at the virtual screen in the simulations have therefore been backscattered by anions in the surface layers rather than being reflected before reaching the surface. The absolute intensity is much lower than in Figs. 2(c) and 2(e).
For the cation-terminated surface, dipoles in the surface layers outside the twin boundaries have an inward rather than an outward component, which traps or slows down electrons and therefore further reduces the measured intensity. For the same physical topography as anion-terminated surfaces, the positive charge of the cation-terminated surface attracts electrons to penetrate the surface where some are trapped or randomly scattered. As a result, the backscattered intensity recorded on the virtual screen is significantly smaller than the reflected intensity.

A (110) model plane is charge neutral [Figs. 4(a) and 4(e)]. The simulations are therefore suitable for comparison with the experimental results obtained from the $\mathrm{CaTiO}_{3}(001)$ surface, which is charge neutral for both possible terminations $(\mathrm{CaO}$ or $\mathrm{TiO}_{2}$ ). The angle between the twin boundary and the surface is approximately $45^{\circ}$ and the ridge or valley angle is smaller than that for a charged surface with $90^{\circ}$ twin wall. Contrast is therefore due to the (weaker) topography or to dipolar scattering. The polarization vectors in the surface layers point outward at one side of the twin boundary, but inward at the other side of the twin boundary [Figs. 4(b) and 4(f)]. When electrons with initial kinetic energy of $0.515 \mathrm{meV}$ approach the surface [Figs. 4(d) and 4(h)], they are attracted by the 

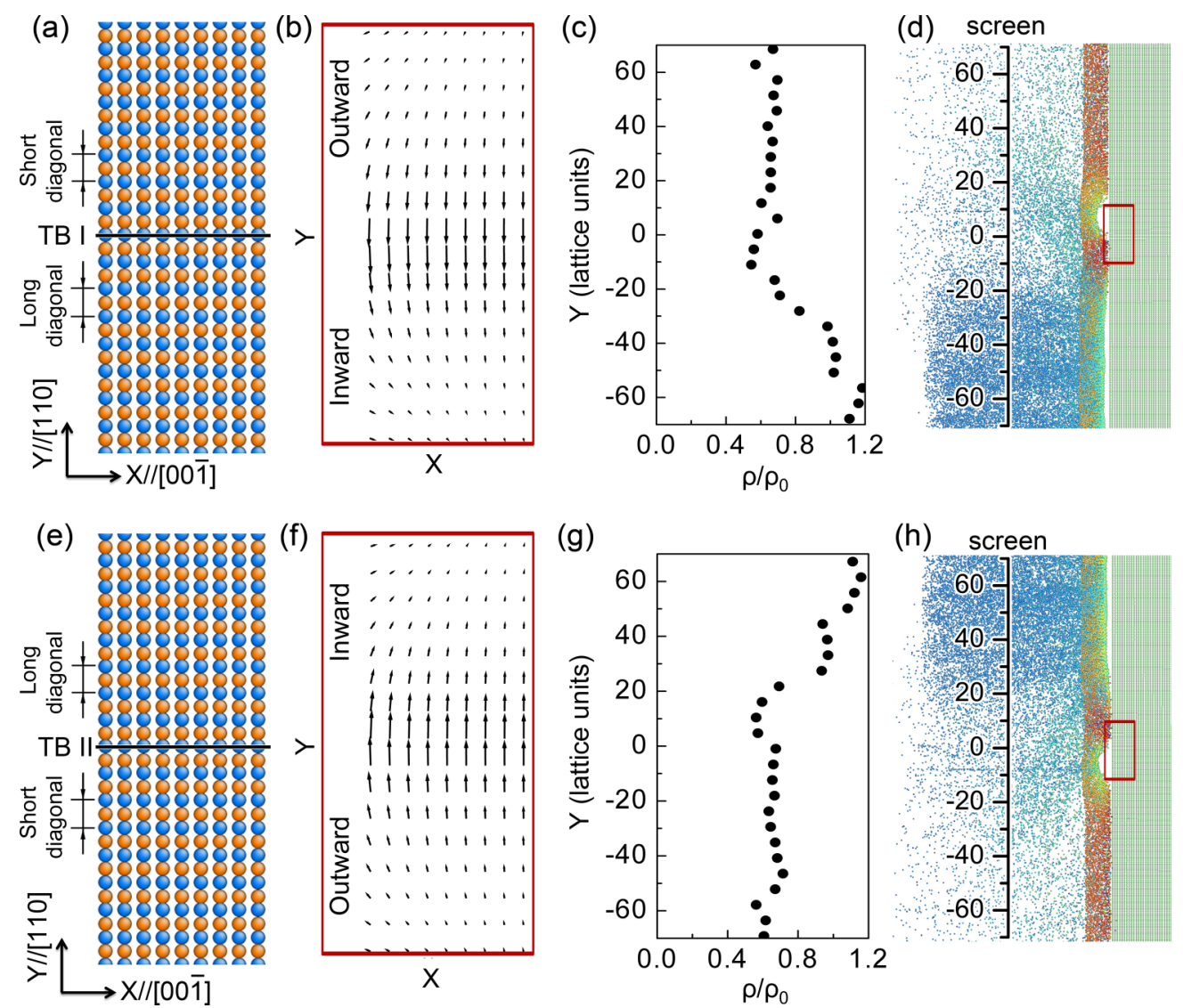

FIG. 5. A configuration with flat neutral surfaces at the intersection with twin boundaries. The simulated 2D configuration is a (1 10$)$ plane in the 3D construction of Fig. 1(b). Enlarged local areas near twin boundary I (TB I) and twin boundary II (TB II) are shown in (a) and (e), respectively. The lattice spacings in $Y$ of the two domains separated by twin boundaries are the long diagonal and short diagonal in Fig. 1(a). The dipole displacements in (b) and (f) are amplified by a factor of 25 for clarity. Other annotations are the same as in Fig. 2.

outward surface polarization and are therefore only partly backscattered. Inward polarization generates a negative surface potential so that the electrons with kinetic energy smaller than the surface potential are reflected, leading to MEM-like images, generating a higher electron density on the virtual screen than LEEM [Figs. 4(c) and 4(g)]. Twin boundary I has outwards polarity, while the polarity in twin boundary II points inwards. Electrons approaching twin boundary I are therefore accelerated more strongly than those reaching the outwards dipoles so that fewer electrons are backscattered, leading to a minimum of electron density in the image of Fig. 4(c). In contrast, at twin boundary II, inward polarity generates negative surface potential so that the electrons are more strongly backscattered at the surface, but topography still diverges the electrons so that the intensity at the twin wall position remains low [Fig. 4(h)].

We finally simulate wall intersections with surfaces where there is no net charge or topography. The contrast stems entirely from dipoles while no ridge or valley structures exist. In this case, we consider a neutral surface with a twin plane located under 45 degrees, as shown in Fig. 5. The simulated $2 \mathrm{D}$ configuration is a (110) plane in the $3 \mathrm{D}$ construction of Fig. 1(b). It contains a $a 1 / a 2$ twin configuration [41] with a flat neutral (001) surface [Figs. 5(a) and 5(e)].

Within our point charge model, the only contrast now stems from the intrinsic dipole moments in the sample. All dipoles are strictly parallel to the surface in the twin boundaries. Outside the twin boundaries, we find vortices with polar rotations which lead to effective polarity perpendicular to the sample surface [Figs. 5(b) and 5(f)]. These polar vectors lead to weak contrast in the MEM simulations [Figs. 5(c) and $5(\mathrm{~g})]$. When the initial kinetic energy of electrons is very low, e.g., $0.13 \mathrm{meV}$ (Fig. 5), the interaction of electrons with the surface is similar to that in Fig. 4. Inward polarity in the surface of the middle domain reflects electrons before they reach the surface; outward surface polarity attracts electrons into the surface leading to lower intensity on the screen. Large in-plane dipoles at the twin boundaries generate effective positive and negative point charges, which attract and reflect electrons more strongly.

We now consider the effect of the initial kinetic energy of the electrons. The anion-terminated surfaces of Fig. 2 systematically lose contrast when the initial kinetic energy of the incoming electrons is increased (Fig. 6). Starting from low energy of $0.515 \mathrm{meV}$, we increase to 33,62 , and 74 $\mathrm{meV}$, and $1.854 \mathrm{eV}$, and observe a transition at a critical value of $62 \mathrm{meV}$. Below the critical value, electrons are reflected before reaching the surface, and the distance between the reflection position and the surface increases with decreasing kinetic energy. Above the critical value, electrons can penetrate the surface and then are backscattered by surface layers. The electron intensity decays with little visible contrast at 

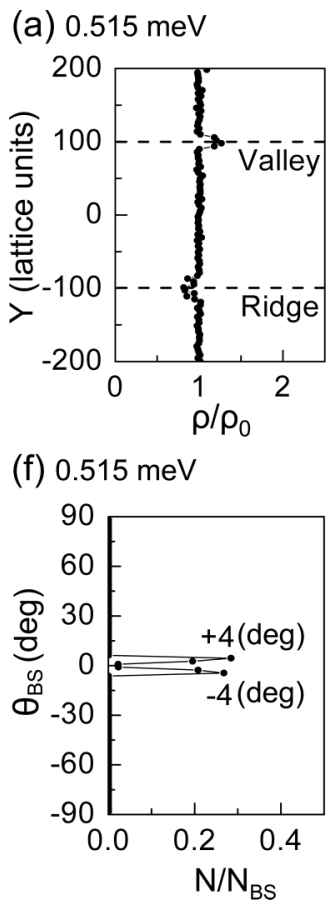

(b) $33 \mathrm{meV}$

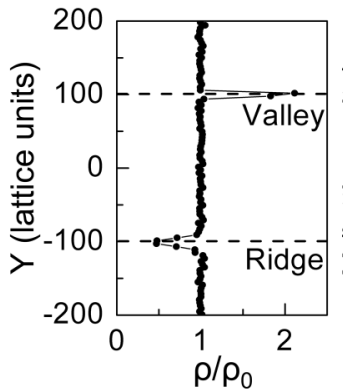

(c) $62 \mathrm{meV}$

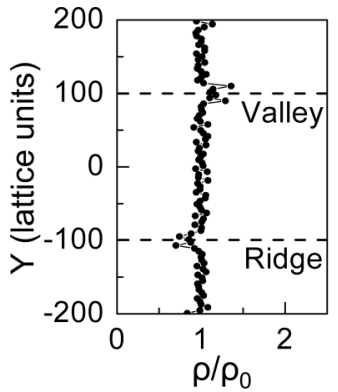

(g) $33 \mathrm{meV}$

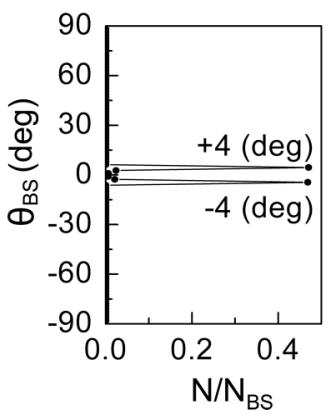

(h) $62 \mathrm{meV}$

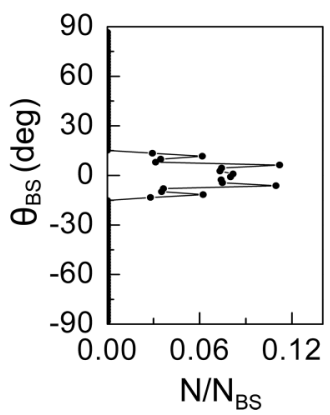

(d) $74 \mathrm{meV}$

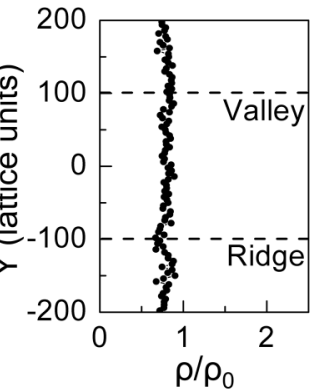

(i) $74 \mathrm{meV}$

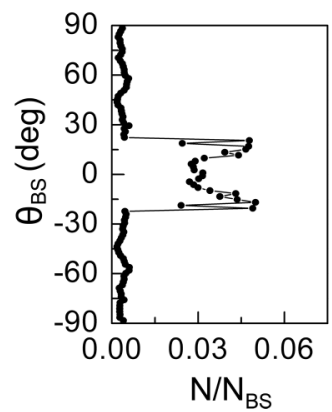

(e) $1.854 \mathrm{eV}$

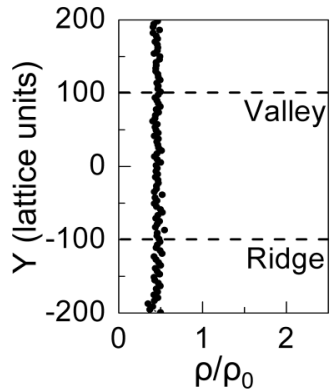

(j) $1.854 \mathrm{eV}$

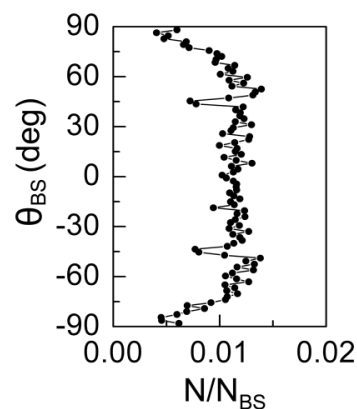

FIG. 6. Kinetic energy dependence on electron backscattering by anion-terminated surfaces (model in Fig. 2). Reduced density of backscattered electron $\left(\rho / \rho_{0}\right)$ at (a) 0.515 , (b) 33,(c) 62, (d) $74 \mathrm{meV}$, and (e) $1.854 \mathrm{eV}$. $\rho_{0}$ is the electron density in the initial configuration; the positions of the twin boundaries are indicated by dashed lines. Their statistics of backscattering angle $\left(\theta_{\mathrm{BS}}\right)$ are shown by (f)-(j). $N_{\mathrm{BS}}$ stands for the total number of backscattered electrons.

$74 \mathrm{meV}$. Figures 6(f)-6(j) show the angular dependence of the reflected or backscattered electrons. At low kinetic energy, the angle histogram shows sharp peaks at $\pm 4^{\circ}$ corresponding to specular reflection from the $2^{\circ}$ twins. At very low kinetic energy $(0.515 \mathrm{meV})$, the peaks are less sharp because reflection occurs further from the surface. As the kinetic energy increases, electrons penetrate and interact more strongly with both subsurface catiions and anions. As a result, the intensity (a) $0.515 \mathrm{meV}$
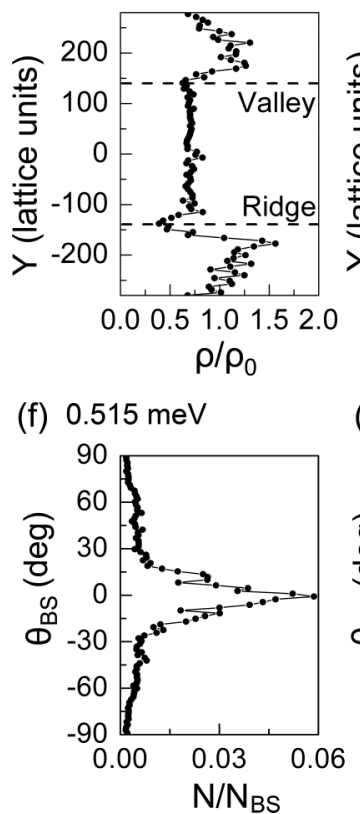

(b) $3.2 \mathrm{meV}$

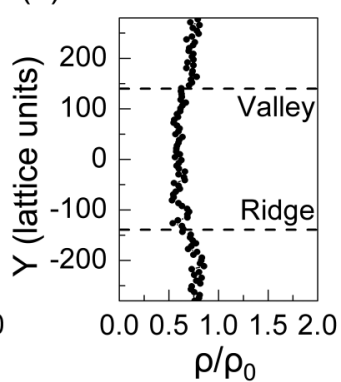

(g) $3.2 \mathrm{meV}$

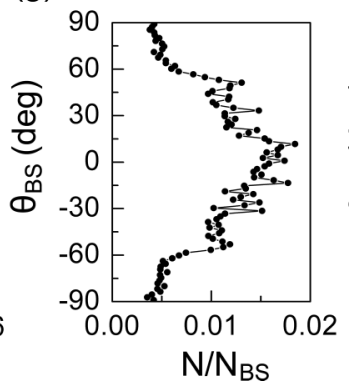

(c) $33 \mathrm{meV}$

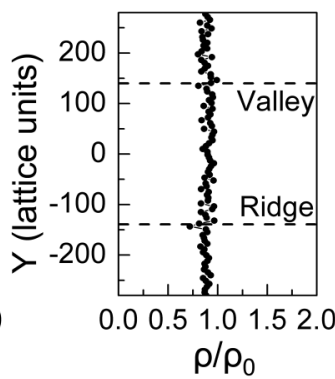

(h) $33 \mathrm{meV}$

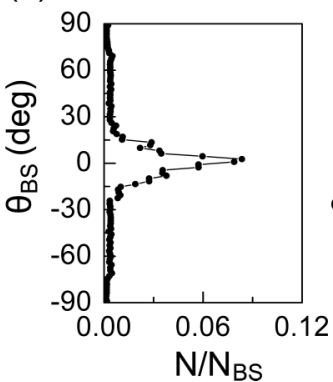

(d) $74 \mathrm{meV}$

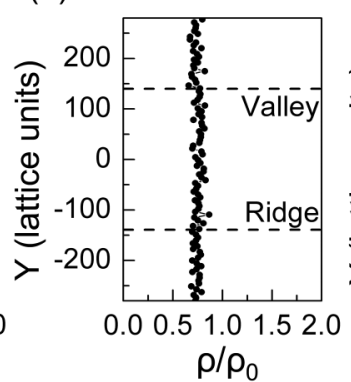

(i) $74 \mathrm{meV}$

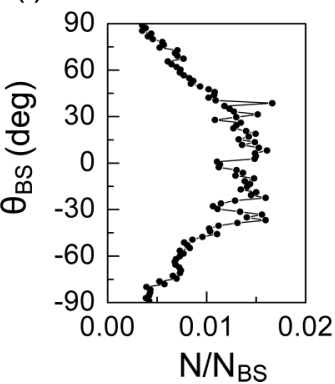

(e) $1.854 \mathrm{eV}$

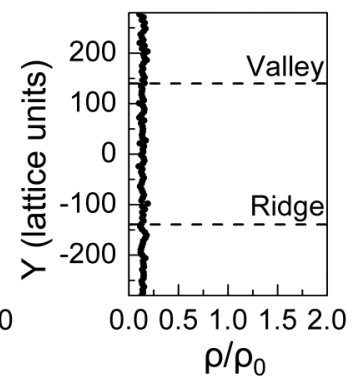

(j) $1.854 \mathrm{eV}$

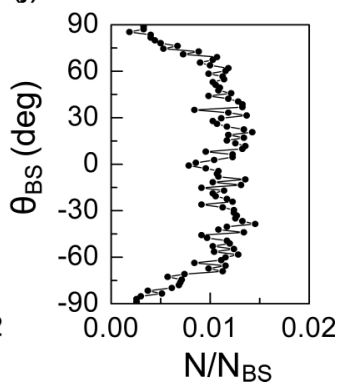

FIG. 7. Kinetic energy dependence on electron backscattering by neutral surfaces (model in Fig. 4). Reduced density of backscattered electron $\left(\rho / \rho_{0}\right)$ at (a) 0.515 , (b) 3.2 , (c) 33 , (d) $74 \mathrm{meV}$, and (e) $1.854 \mathrm{eV}$. $\rho_{0}$ is the electron density in the initial configuration; the positions of twin boundaries are indicated by dashed lines. Their statistics of backscattering angle $\left(\theta_{\mathrm{BS}}\right)$ are shown by (b)-(f). $N_{\mathrm{BS}}$ stands for the total number of backscattered electrons. 

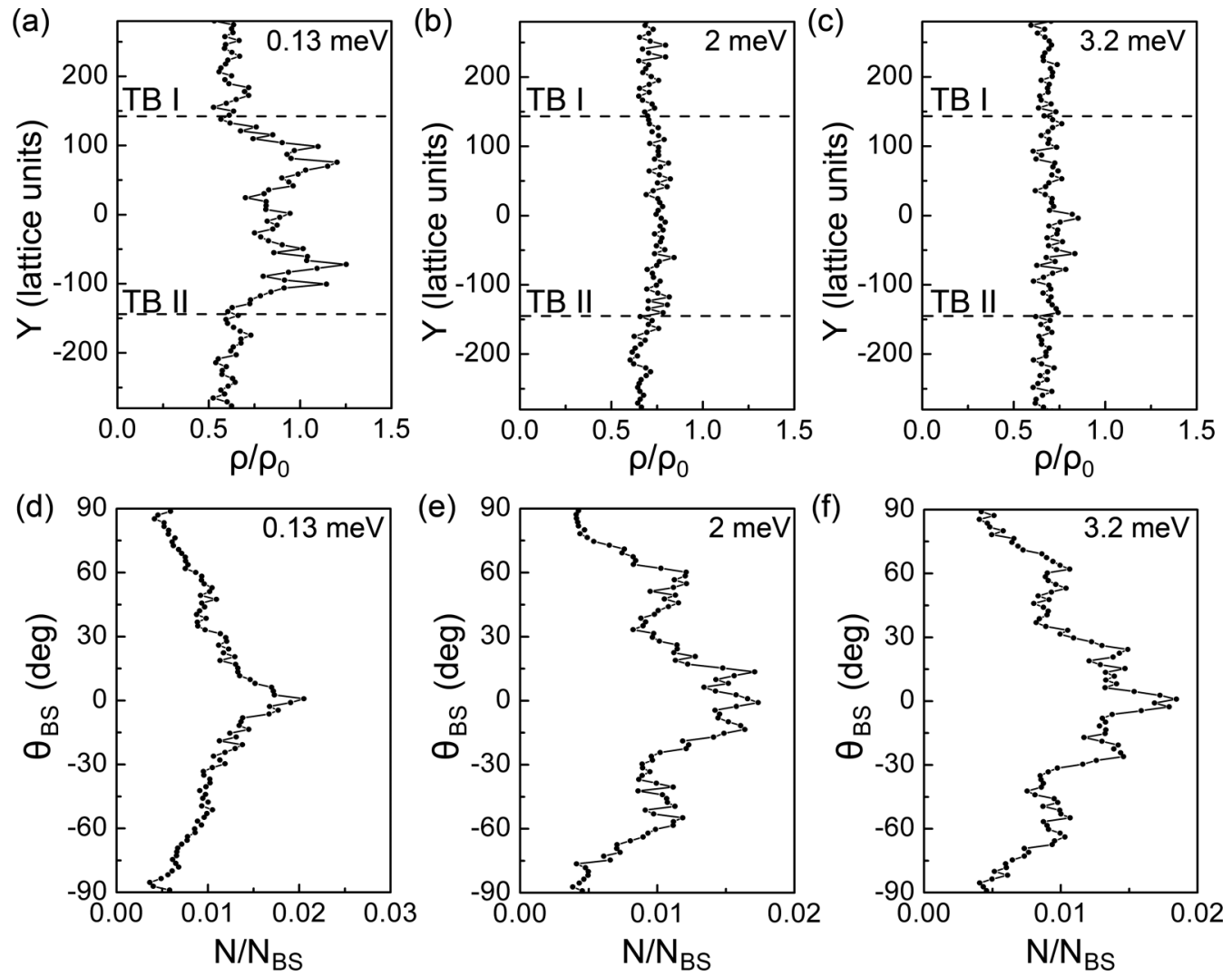

FIG. 8. Kinetic energy dependence on electron backscattering by neutral flat surfaces (model in Fig. 5). Reduced density of backscattered electron $\left(\rho / \rho_{0}\right)$ at (a) 0.13 , (b) 2.00 , and (c) $3.20 \mathrm{meV}$. The electron density $\left(\rho / \rho_{0}\right)$ from scattering by the middle domain is higher and caused by inward polarity. Outward polarity in the top and bottom regions results in lower density. The contrast decreases with increasing kinetic energy. The statistics of the backscattering angle $\left(\theta_{\mathrm{BS}}\right)$ are shown in (d)-(f). $\rho_{0}$ is the electron density in the initial configuration, $N_{\mathrm{BS}}$ stands for the total number of backscattered electrons.

maxima in Figs. 6(a)-6(e) are strongly attenuated and the angular distribution broadens due to multiple electron-ion interactions. At the same time, the deeper the electrons penetrate into the sample, the less they are sensitive to variations in the surface potential due to the twin walls. The angular dependence of the scattered electrons still shows peaks at $+4^{\circ}$ and $-4^{\circ}$ for energy below the critical value, while even this contrast disappears at $1.854 \mathrm{eV}$.

Positively charged surfaces show no significant contrast and increasing the initial kinetic energy of the electrons does not change this result. This is because our model is limited to electron-ion Coulomb interaction and the MD relaxation of the slab does not include a calculation of the work function in relation to the initial electron energy.

Neutral surfaces show initial steps in the intensity at twin boundaries [Fig. 7(a)], the height of which decreases rapidly when the initial kinetic energy increases to $3.2 \mathrm{meV}$ [Fig. 7(b)]. The intensity distribution across the twins at $0.515 \mathrm{meV}$ kinetic energy is the result of weaker physical topography and the difference in domain surface polarity on either side of the twin. The latter implies the existence of a stray field deviating the reflected electrons laterally, along $y$. The asymmetric intensity profile across the twin reflects this. At slightly higher energy $(3.2 \mathrm{meV}), 70 \%$ of all electrons are reflected but the contrast is lower because the reflection occurs deeper in the lattice. At $1.854 \mathrm{eV}$, all contrast disappears and only $14 \%$ of the electrons are reflected.

The kinetic energy dependence of the electron backscattering from flat neutral surfaces (model in Fig. 5) is shown in Fig. 8. At low kinetic energy there is a step in intensity at the twin boundary due to the stray lateral field induced by the twin wall polarization parallel to the surface. When we increase the energy from an initial value of $0.13 \mathrm{meV}$ to higher values, the contrast becomes weaker and disappears at
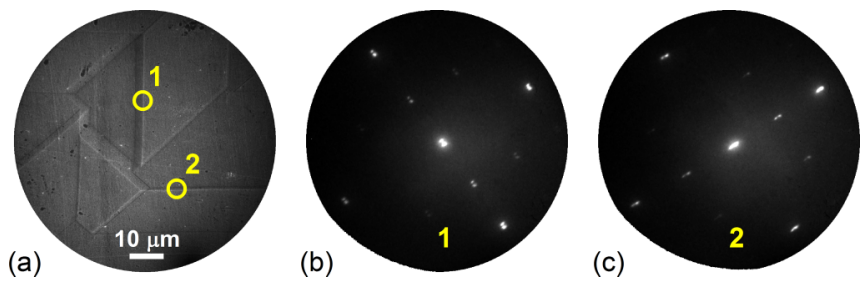

FIG. 9. MEM image (field of view $75 \mu \mathrm{m}$ ) of another $\mathrm{CaTiO}_{3}$ (001) surface at a start voltage of $-0.13 \mathrm{eV}$, showing twin walls. No significant domain contrast is observed. (b), (c) LEED images at $17 \mathrm{eV}$ acquired from regions straddling the horizontal and vertical twins in (a), defined by positioning an appropriate field aperture. The double LEED spots directly reflect the twinning. 

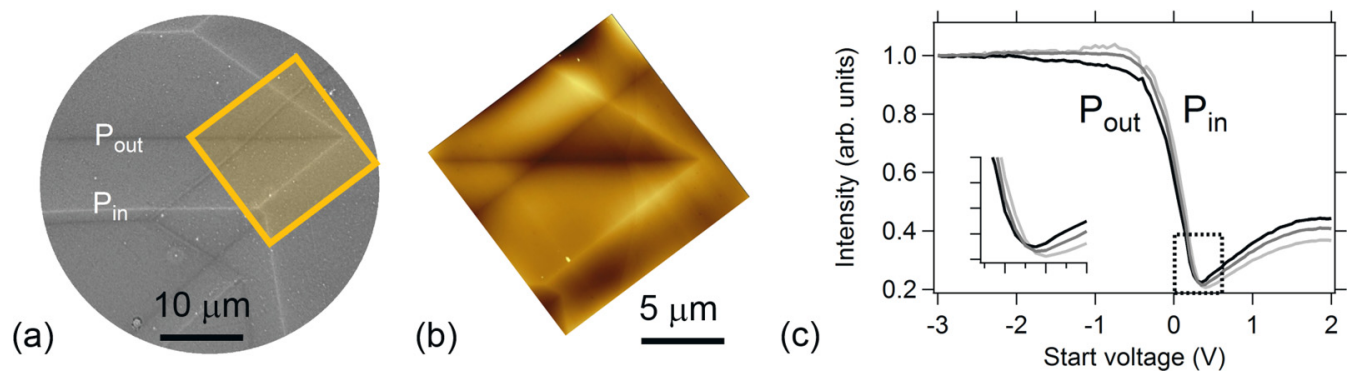

FIG. 10. (a) MEM image of the $\mathrm{CaTiO}_{3}$ (111) surface at a start voltage $-0.6 \mathrm{~V}$ showing bright and dark domain walls. Far from the domain wall there is no surface potential contrast between domains. The domain walls are either dark or bright, reflecting positive or negative surface topological charge (upward- or downwards-pointing polarity). The orange square is the zone analyzed by AFM. (b) AFM image of part of (a) showing the surface topography in perspective. The maximum topography dynamic is $40 \mathrm{~nm}$. (c). Electron intensity as a function of start voltage at outward (black), $P_{\text {out }}$, inward (light gray), $P_{\text {in }}$, polarity twins and at domain surfaces (gray). The $P_{\text {out }}$ and $P_{\text {in }}$ curves are measured at the walls as indicated in (a). The inset illustrates the small, $50 \mathrm{mV}$ shift in surface potential between outward and inward polarity twins.

$3.2 \mathrm{meV}$. Interestingly, the angular distribution is peaked at an initial kinetic energy of $2 \mathrm{meV}$, presumably because the surface potential as seen by the incoming electrons is flatter at very low kinetic energy.

\section{B. Experiment}

We compare the simulations with experimental results on $\mathrm{CaTiO}_{3}$ (001) and (111). While the interactions in the simulations relate to generic toy models, insights may be gained into the symmetry of the pattern formation and the various types of surface scattering. The (001) surface is $\mathrm{CaO}$ or $\mathrm{TiO}_{2}$ terminated and therefore charge neutral and can be compared with the MEM-LEEM images of the (110) or (001) model surfaces, i.e., Figs. 4, 5 and 7, 8. The (111) termination, on the other hand, has either a negatively $\left(\mathrm{CaO}_{3}^{4-}\right)$ or positively $\left(\mathrm{Ti}^{4+}\right)$ charged surface layer and is compared to the experimental results for $\mathrm{CaTiO}_{3}$ (111) with the anion- and cation-terminated (100) model surfaces, respectively.

The experimental $\mathrm{CaTiO}_{3}$ surfaces show twins with inwards/outwards polarities, ridges/valleys, and even electron intensity contrast between adjacent domains. We will consider three distinct results showing the role of topography, twin wall polarity without surface dipole contrast, and surface dipoles with additional surface dipole contrast.

\section{Physical topography}

The characteristic physical topography of the twin/surface intersection is revealed by low-energy diffraction from microscopic regions including both sides of vertical and horizontal twin boundaries. Figure 9 shows a MEM image of a $\mathrm{CaTiO}_{3}$ (001) sample. The twin walls are horizontal, vertical, and at $45^{\circ}$ in the image. There is also slight domain contrast between adjacent domains. Figures 9(b) and 9(c) show LEED images recorded at $17 \mathrm{eV}$ electron energy from regions 1 and 2 , defined by a circular field aperture in an image plane of the microscope. The splitting of the $1 \times 1$ LEED spots about symmetry axes confirms the twin nature of the walls in Fig. 9(a) and, furthermore, the angular splitting corresponds to a $1^{\circ}$ twin angle. We can therefore compare this surface to the simulations in which the role of physical topography was predicted to focus (valleys) or defocus (ridges) the reflected electrons giving rise to specular peaks in the angular distributions.

\section{Domain-wall polarity}

Figure 10(a) shows a MEM image of the $\mathrm{CaTiO}_{3}$ (111) surface acquired at a start voltage $-0.6 \mathrm{~V}$ in a field of view of $75 \mu \mathrm{m}$ and at a temperature of $300{ }^{\circ} \mathrm{C}$. The twin boundaries are dark or bright straight lines with sharp contrast with respect to the domain surfaces. Far from the twin walls, there is no observable contrast between adjacent domains. Horizontal walls are alternately bright and dark, suggesting a typical factory rooflike topography.

The sharp contrast between twin walls and domains is similar to the intensity distributions obtained in Figs. 2(c) and $2(\mathrm{~g})$. The start voltage for the image in Fig. 10(a) is below the MEM-LEEM transition, and therefore bright intensity corresponds to inwards polarity $\left(P_{\text {in }}\right)$ which provides a negative shift to the surface potential, enhancing the electron reflectivity, whereas dark intensity means lower electron reflectivity and therefore outwards polarity $\left(P_{\text {out }}\right)$. The atomic force microscopy (AFM) topography in Fig. 10(b) confirms that the ridges provide bright contrast and the valleys dark contrast in the electron image. At first sight, this is surprising because it is the opposite of the simulation contrast for the anion-terminated surface. However, the simulated electron intensity distribution is recorded on a virtual screen 50 lattice constants from the surface without using any lenses. In the experimental microscope, there are three converging lenses; as a result, the contrast predicted by the simulations should be inverted [35] with downward-pointing polarity and ridge topography that will appear bright and an upward-pointing polarity twin in a valley topography that will appear dark. The simulations thus become fully consistent with the experimental results. Both anion- and cation-terminated surfaces should show contrast due to the potential difference between the polar wall visible in Figs. 3(b) and 3(f) and the surrounding weaker dipolar surfaces. Figure 10(c) shows the electron intensity as a function of start voltage at outward (black), $P_{\text {out }}$, inward (light gray), $P_{\text {in }}$, polarity twins, and at domain surfaces far from the walls (gray). The curve obtained far from the domain walls is the same in all domains, suggesting identical surface charge and polarity. The inset to Fig. 10(c) illustrates the $50 \mathrm{mV}$ shift in surface potential between outward and inward polarity 


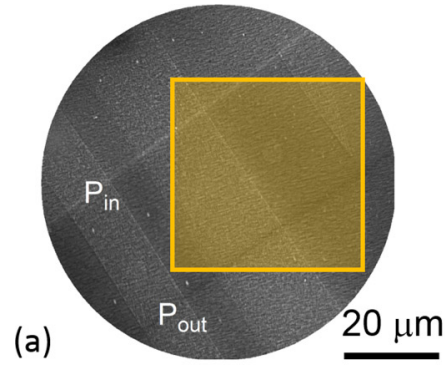

(b)

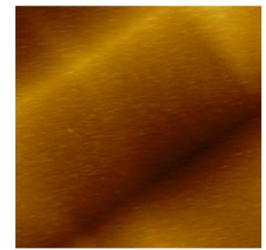

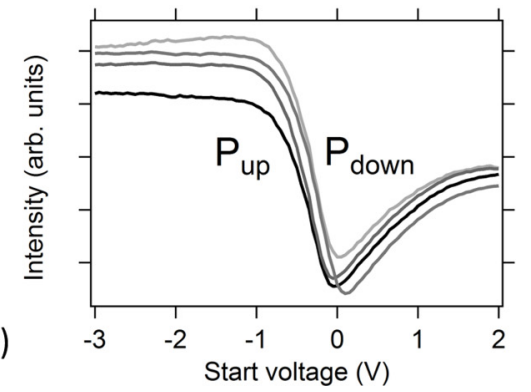

FIG. 11. (a) MEM image of $\mathrm{CaTiO}_{3}(001)$ surface (sample SurfaceNet $\mathrm{GmbH}$ ) at a start voltge $-0.4 \mathrm{~V}$. Outward and inward polarity twins are indexed $P_{\text {out }}$ and $P_{\text {in }}$. Contrast is also visible between adjacent domains. (b) AFM topography of the orange region in (a) shows the ridge-valley structure. (c) Electron intensity as a function of start voltage measured at $P_{\text {out }}$ and $P_{\text {in }}$ twins (black and light gray curves, respectively) and from two adjacent domains (gray). Contrast is visible in all cases; the MEM-LEEM transition shift between the $P_{\text {out }}$ and $P_{\text {in }}$ curves is $150 \mathrm{mV}$.

twins, confirming that twin wall polarity does indeed induce a local variation in surface potential. On the other hand, the surface potential as deduced from the MEM-LEEM transition for adjacent domains is identical, suggesting identical surface termination and dipoles, just as in Fig. 2 or Fig. 3.

\section{Wall and surface dipoles}

Finally, in Fig. 11, we show the MEM image of a commercial $\mathrm{CaTiO}_{3}(001)$ acquired at $-0.4 \mathrm{~V}$ start voltage. The bright and dark contrast due to the opposite polarities of the twin walls (outward and inward polarity twins are indexed $P_{\text {out }}$ and $\left.P_{\text {in }}\right)$ is visible along high-symmetry directions, but in addition there is clear contrast between intensity from adjacent domains. The ridge-valley structure is confirmed by the AFM topography scan shown in Fig. 11(b). The electron intensities as a function of start voltage recorded at $P_{\text {out }}$ and $P_{\text {in }}$ twins and from two adjacent domains are shown in Fig. 11(c). The surface potential of the twin walls differs by $150 \mathrm{mV}$. The values straddle the surface potential values of adjacent domains which show a contrast of 50-100 mV.

Contrary to the $\mathrm{CaTiO}_{3}$ (111) surface but similar to the case of the charge neutral surface simulated in Fig. 4, both topography and surface polarity create contrast in MEM. The valley-ridge structure and the presence of surface polarity give rise to domain contrast on either side of the twins as well as twin walls with opposite, out-of-plane polarity.

\section{DISCUSSION}

We have investigated the surface polarity near the intersection of surface and twin boundaries using a simple mechanical model coupling anion and cation sublattices. We confirm that strain relaxations near perpendicular intersections are large and follow the patterns predicted by Refs. [27,28]. The strain profiles imply local elastic softening at the intersection point of twin and surface. Next to these "soft spots," we confirm local ridges where the surface layer bulges outwards. This effect should not be confused with the ridges and valleys of the twin structure, which relate to the deformation of the surface by the geometric shear of the twin angle. The strain fields then induce polarity, as a direct consequence of the Landau springs and the anion-cation coupling of our simple toy model [29]. Surface polarity was more generally predicted in Ref. [42] as related to the inevitable strain gradients perpendicular to the surface layer [43]. In our simulations, however, in one case the surface polarity is the same on both sides of the twin wall (Figs. 2 and 3), whereas in the case of twins at $45^{\circ}$ with respect to the neutral surface, it changes sign. The AFM results confirm the shallower ridge and valley topography of the latter. It appears that the dipole-dipole interaction between the dipoles in the $45^{\circ}$ twins and the surface dipoles plays a role in defining antiparallel surface polarity on either side of the twin wall.

For all configurations, we find that the surfaces are polar and that this polarity changes dramatically near the intersection with the twin wall. The simulated dipolar patterns are shown in Fig. 12, with the profiles for anion-terminated surfaces with perpendicular twin boundaries. The two distinct configurations relate to a valley domain wall [Fig. 12(a)] and a ridge domain wall [Fig. 12(b)]. The polarization inside the twin boundary $P_{0}=1.6 \times 10^{-19} \mathrm{C} \times 3 \mathrm{pm} /\left(1 \times 10^{-10} \mathrm{~m}\right)^{3}=$ $0.48 \mathrm{C} / \mathrm{m}^{2}$ for a maximum displacement of $3 \mathrm{pm}$ in a volume
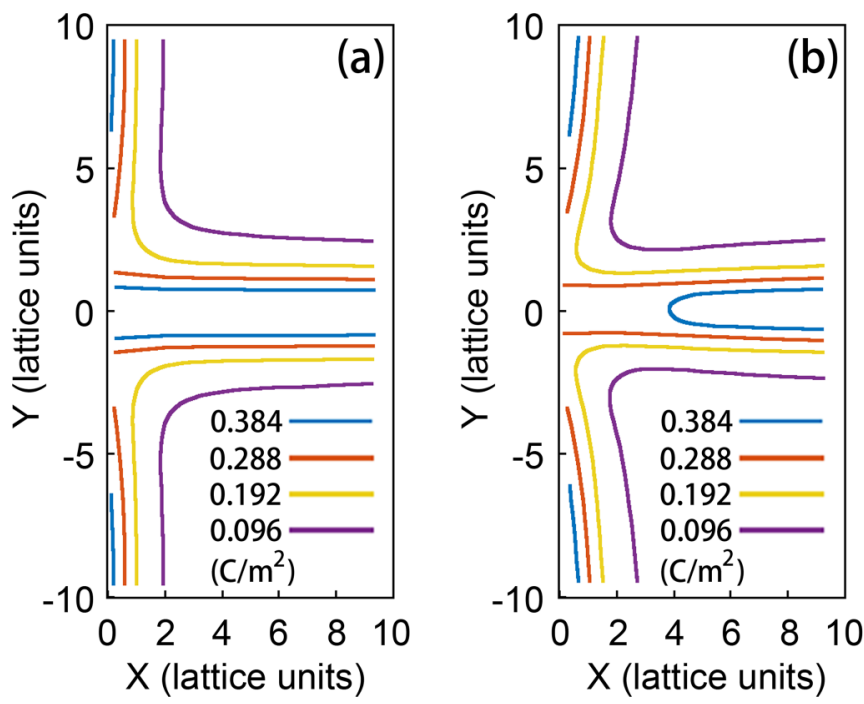

FIG. 12. Distribution of the polarization magnitude $|P|$ in surface layers near intersections with twin boundaries (the polarization inside the twin boundary $P_{0}=0.48 \mathrm{C} / \mathrm{m}^{2}$ ). The surfaces are anion terminated. $X$ and $Y$ represent the distance from the left surface and the twin boundary. The topology relates to (a) a valley and (b) a ridge. 
of $1 \AA^{3}$. A typical experimental value is $P_{0}=0.2 \mathrm{C} / \mathrm{m}^{2}$ in the domain wall of $\mathrm{CaTiO}_{3}$ [3]. We note that this value is similar to the spontaneous bulk polarization in $\mathrm{BaTiO}_{3}$ [44].

We identified three major effects due to the (1) surface termination (i.e., charge), (2) geometry of the surface (ridges and valleys), and (3) surface dipoles (polarity). All other features more than three layers beneath the surface are effectively screened and cannot be observed. In our simulations, the density of reflected electrons at the virtual screen is mostly influenced by surface charges, reproducing how local work function variations can influence the electron reflectivity. Inclined surfaces scatter the beam preferentially in the direction of the inclination. When two surfaces form a valley, their scattering superimposes and is hence enhanced near the intersection with the domain boundary. If the surfaces form a ridge, the two beams diverge and reduce the overlap. The resulting LEEM/MEM pattern shows dark patterns in the first case and light patterns in the second. In our model, the absence of a net surface charge allows polarity due to the surface dipoles to play a dominant role in determining the electron reflectivity. In the case of $45^{\circ}$ boundaries, they generate patterns with dark and light lines combined with a strong change of LEEM intensity between the adjacent domains. The third case considered is $45^{\circ}$ twins with no surface topography, giving rise to inplane polarization. The stray field laterally deviates electrons and gives rise to an asymmetric intensity profile in MEM perpendicular to the wall and the appearance of vortexlike polarization structures on either side of the twin wall.

The main limit of the model is that electron scattering is mainly from the anion sublattice, and thus the simulations cannot model reflection at the cation-terminated surfaces for very low initial electron energy. Instead, electrons are always attracted into the surface by the positive point charges and are backscattered by the first few anion-containing layers. The contrary is the case for the anion-terminated surface from which electrons are reflected before reaching the surface. However, in the case of the anion termination, for sufficiently high initial electron energy ( $74 \mathrm{meV}$ in the simulations), electrons do penetrate before undergoing single or multiple scattering from anions in deeper layers or are trapped by interaction with subsurface cations. The twin angle is implicitly included in the molecular dynamics calculations of the electron scattering. The model can therefore simulate the effect of surface charge, topography, and polarity and provide insight into how the three combine to produce contrast in MEM-LEEM experiments on ferroelastic surfaces.

\section{ACKNOWLEDGMENTS}

Z.Z. is grateful for the financial support by the National Natural Science Foundation of China (Grant No. 51602255), the Programs of Key Research and Development Plan of Shaanxi Province (Grant No. 2018ZDXM-GY-145), the Scientific Research Program of Shaanxi Education Department (Grant No. 16JK1561), and the Doctoral Starting Fund of Xi'an University of Technology (Grant No. 101-451115016). E.K.H.S. is grateful to the Leverhulme Trust (Grant No. EM-2016-004) and EPSRC (Grant No. EP/K009702/1). This work is supported by a public grant overseen by the French National Research Agency (ANR) as part of the Investissements d'Avenir program (Labex NanoSaclay, Ref. No. ANR10-LABX-0035) and the DFG-ANR 2014 project HREELM (ANR-14-CE35-0019-03).
[1] E. K. H. Salje, Annu. Rev. Mater. Res. 42, 265 (2012).

[2] K. Alexander, L. E. Cross, and J. Fousek, Domains in Ferroic Crystals and Thin Films (Springer, New York, 2010).

[3] S. Van Aert, S. Turner, R. Delville, D. Schryvers, G. Van Tendeloo, and E. K. H. Salje, Adv. Mater. 24, 523 (2012).

[4] P. Zubko, G. Catalan, and A. K. Tagantsev, Annu. Rev. Mater. Res. 43, 387 (2013).

[5] S. A. Hayward, J. Chrosch, E. K. H. Salje, and M. A. Carpenter, Eur. J. Mineral. 8, 1301 (1996).

[6] J. Chrosch and E. K. H. Salje, J. Appl. Phys. 85, 722 (1999).

[7] M. Calleja, M. T. Dove, and E. K. H. Salje, J. Phys.: Condens. Matter 15, 2301 (2003).

[8] C.-L. Jia, K. W. Urban, M. Alexe, D. Hesse, and I. Vrejoiu, Science 331, 1420 (2011).

[9] D. D. Fong, A. M. Kolpak, J. A. Eastman, S. K. Streiffer, P. H. Fuoss, G. B. Stephenson, C. Thompson, D. M. Kim, K. J. Choi, C. B. Eom, I. Grinberg, and A. M. Rappe, Phys. Rev. Lett. 96, 127601 (2006).

[10] P. S. Bednyakov, T. Sluka, A. K. Tagantsev, D. Damjanovic, and N. Setter, Sci. Rep. 5, 15819 (2015).

[11] Y. Watanabe, Phys. Rev. B 59, 11257 (1999).

[12] E. Salje and H. Zhang, Phase Transit. 82, 452 (2009).

[13] D. D. Viehland and E. K. H. Salje, Adv. Phys. 63, 267 (2014).
[14] H. Yokota, H. Usami, R. Haumont, P. Hicher, J. Kaneshiro, E. K. H. Salje, and Y. Uesu, Phys. Rev. B 89, 144109 (2014).

[15] L. Goncalves-Ferreira, S. A. T. Redfern, E. Artacho, and E. K. H. Salje, Phys. Rev. Lett. 101, 097602 (2008).

[16] J. F. Scott, E. K. H. Salje, and M. A. Carpenter, Phys. Rev. Lett. 109, 187601 (2012).

[17] H. Yokota, S. Matsumoto, E. K. H. Salje, and Y. Uesu, Phys. Rev. B 98, 104105 (2018).

[18] Y. Kim, M. Alexe, and E. K. H. Salje, Appl. Phys. Lett. 96, 032904 (2010).

[19] A. Aird and E. K. H. Salje, J. Phys.: Condens. Matter 10, L377 (1998).

[20] S. Conti, S. Muller, A. Poliakovsky, and E. K. H. Salje, J. Phys.: Condens. Matter 23, 142203 (2011).

[21] B. Houchmandzadeh, J. Lajzerowicz, and E. Salje, J. Phys.: Condens. Matter 3, 5163 (1991).

[22] T. Zykova-Timan and E. K. H. Salje, Appl. Phys. Lett. 104, 082907 (2014).

[23] R. I. Eglitis and D. Vanderbilt, Phys. Rev. B 77, 195408 (2008).

[24] E. Heifets, E. A. Kotomin, and J. Maier, Surf. Sci. 462, 19 (2000).

[25] R. I. Eglitis, Phys. Status Solidi B-Basic Solid State Phys. 252, 635 (2015).

[26] R. I. Eglitis, Ferroelectrics 483, 53 (2015). 
[27] J. Novak and E. K. H. Salje, Eur. Phys. J. B 4, 279 (1998).

[28] J. Novak and E. K. H. Salje, J. Phys.: Condens. Matter 10, L359 (1998).

[29] E. K. H. Salje, S. Z. Li, M. Stengel, P. Gumbsch, and X. D. Ding, Phys. Rev. B 94, 024114 (2016).

[30] G. F. Nataf, M. Guennou, J. Kreisel, P. Hicher, R. Haumont, O. Aktas, E. K. H. Salje, L. Tortech, C. Mathieu, D. Martinotti, and N. Barrett, Phys. Rev. Mater. 1, 074410 (2017).

[31] N. Barrett, J. E. Rault, J. L. Wang, C. Mathieu, A. Locatelli, T. O. Mentes, M. A. Nino, S. Fusil, M. Bibes, A. Barthelemy, D. Sando, W. Ren, S. Prosandeev, L. Bellaiche, B. Vilquin, A. Petraru, I. P. Krug, and C. M. Schneider, J. Appl. Phys. 113, 187217 (2013).

[32] N. Barrett, J. Dionot, D. Martinotti, E. K. H. Salje, and C. Mathieu, Appl. Phys. Lett. 113, 022901 (2018).

[33] O. Copie, N. Chevalier, G. Le Rhun, C. L. Rountree, D. Martinotti, S. Gonzalez, C. Mathieu, O. Renault, and N. Barrett, ACS Appl. Mater. Inter. 9, 29311 (2017).
[34] G. F. Nataf, P. Grysan, M. Guennou, J. Kreisel, D. Martinotti, C. L. Rountree, C. Mathieu, and N. Barrett, Sci. Rep. 6, 33098 (2016).

[35] S. A. Nepijko, N. N. Sedov, and G. Schonhense, J. Microsc. 203, 269 (2001).

[36] Z. Zhao, X. Ding, and E. K. H. Salje, Appl. Phys. Lett. 105, 112906 (2014).

[37] S. Plimpton, J. Comput. Phys. 117, 1 (1995).

[38] S. Nose, Mol. Phys. 57, 187 (1986).

[39] W. G. Hoover, Phys. Rev. A 31, 1695 (1985).

[40] J. Li, Model. Simul. Mater. Sci. Eng. 11, 173 (2003).

[41] B. Casals, A. Schiaffino, A. Casiraghi, S. J. Hamalainen, D. Lopez Gonzalez, S. van Dijken, M. Stengel, and G. Herranz, Phys. Rev. Lett. 120, 217601 (2018).

[42] A. K. Tagantsev and A. S. Yurkov, J. Appl. Phys. 112, 044103 (2012).

[43] E. K. H. Salje, J. Phys.: Condens. Matter 20, 485003 (2008).

[44] J. B. Neaton and K. M. Rabe, Appl. Phys. Lett. 82, 1586 (2003). 